\title{
Synergies between ELTs and the SKA
}

\author{
Steve Rawlings \\ Astrophysics, Oxford University, UK
}

\begin{abstract}
Over the coming two decades astronomers will plan, construct and exploit extremely large telescopes operating at both optical (ELTs) and radio (SKA) wavelengths. In studies of galaxy evolution and cosmology the combination of the two types of facility are likely to be extremely powerful. In this paper I will review the key questions that the ELT/SKA combination seem set to solve, focussing on the unique advantages afforded by optical and radio observations against the backdrop of the other theoretical and observational information to be available by 2020 .
\end{abstract}

